\title{
Confiabilidade intra e interexaminadores do paquímetro nos movimentos
}

\section{mandibulares}

\author{
Intra and interobserver reliability of the caliper jaw movements \\ Fiabilidad intra e interexaminador de la pinza en los movimientos mandibulares
}

\author{
Juliana Silva Costa \\ ORCID: https://orcid.org/0000-0003-3522-1260 \\ Universidade Federal de Sergipe, Brasil \\ E-mail: fisio.jucosta@gmail.com \\ Fernanda Paixão Amado \\ ORCID: https://orcid.org/0000-0002-5837-1408 \\ Universidade Federal de Sergipe, Brasil \\ E-mail: passionfernanda@gmail.com \\ Johnatan Weslley Araujo Cruz \\ ORCID: https://orcid.org/0000-0002-5464-4114 \\ Universidade Federal de Sergipe, Brasil \\ E-mail: johnweslley2@gmail.com \\ Jessica Paloma Rosa Silva \\ ORCID: https://orcid.org/0000-0002-8235-1255 \\ Universidade Federal de Sergipe, Brasil \\ E-mail: jpalomarosa@gmail.com \\ Leonardo Yung dos Santos Maciel \\ ORCID: https://orcid.org/0000-0001-5381-8015 \\ Universidade Federal de Sergipe, Brasil \\ E-mail: yung_maciel@ hotmail.com \\ Marcela Ralin de Carvalho Deda Costa \\ ORCID: https://orcid.org/0000-0003-1705-4833 \\ Universidade Federal de Sergipe, Brasil \\ E-mail: deda.marcela@hotmail.com
}

\begin{abstract}
Resumo
Introdução: A análise das limitações dos movimentos mandibulares representa um importante sinal para a compreensão do diagnóstico de alterações funcionais do sistema estomatognático. Atualmente um dos principais instrumentos utilizados por fisioterapeutas, odontólogos e fonoaudiólogos para avaliação e mensuração dos movimentos mandibulares é o paquímetro, um instrumento não invasivo e de fácil utilização que oferece medidas objetivas na avaliação do sistema estomatognático. Objetivo: Avaliar a confiabilidade intra e interexaminadores da avaliação dos movimentos mandibulares por meio do paquímetro. Métodos: Trata-se de um estudo com delineamento transversal e de caráter comparativo realizado em indivíduos entre 18 e 25 anos com grau leve ou sem sintomas de DTM de acordo com o ProDTMMulti. A coleta foi realizada em dois dias de mensuração dos movimentos mandibulares de abertura, protrusão, lateralidade direita e esquerda para cada examinado por meio do paquímetro analógico de plástico. A análise de concordância foi calculada pelo Coeficiente de Correlação Intraclasse (ICC), onde foi adotado um nível de significância de 5\%. Resultados e Discussão: O paquímetro é um instrumento confiável e apresenta uma excelente confiabilidade intraexaminador para quase todos os movimentos, exceto desvio lateral esquerdo que demonstrou uma moderada confiabilidade e a confiabilidade interexaminadores foi moderada para maioria dos movimentos. Conclusão: Pode-se concluir que o paquímetro é um método confiável para analises intraexaminadores, no entanto, isso não ocorre para avaliações inter-examinadores, onde apresenta confiabilidade moderada, além de apresentar grande utilidade para a aplicação clínica que envolva um ou mais examinadores durante o atendimento.
\end{abstract}

Palavras-chave: Síndrome da disfunção da articulação temporomandibular; Reprodutibilidade dos testes; Avaliação.

\begin{abstract}
Introduction: The analysis of mandibular movement limitations represents an important sign for understanding the diagnosis of functional alterations in the stomatognathic system. Currently, one of the main instruments used by physiotherapists, dentists and speech therapists to assess and measure mandibular movements is the caliper, a noninvasive and easy-to-use instrument that offers objective measurements in the assessment of the stomatognathic system. Objective: To assess the intra- and inter-examiner reliability of the assessment of mandibular movements using the caliper. Methods: This is a cross-sectional and comparative study carried out in individuals between 18 and
\end{abstract}


25 years old with mild or no symptoms of TMD according to the ProDTMMulti. The collection was carried out in two days of measuring the mandibular movements of opening, protrusion, right and left laterality for each examined by means of an analog plastic caliper. The agreement analysis was calculated using the Intraclass Correlation Coefficient (ICC), where a significance level of 5\% was adopted. Results and Discussion: The caliper is a reliable instrument and has excellent intra-examiner reliability for almost all movements, except for left lateral deviation, which demonstrated moderate reliability and the inter-examiner reliability was moderate for most movements. Conclusion: It can be concluded that the caliper is a reliable method for intra-examiner analyses, however, this does not occur for interexaminer assessments, where it has moderate reliability, in addition to being very useful for clinical application involving one or more examiners during the service.

Keywords: Temporomandibular joint dysfunction syndrome; Reproducibility of results; Evaluation.

\section{Resumen}

Introducción: El análisis de las limitaciones del movimiento mandibular representa un signo importante para comprender el diagnóstico de alteraciones funcionales en el sistema estomatognático. Actualmente, uno de los principales instrumentos utilizados por fisioterapeutas, dentistas y logopedas para evaluar y medir los movimientos mandibulares es el calibre, un instrumento no invasivo y fácil de usar que ofrece medidas objetivas en la valoración del sistema estomatognático. Objetivo: Evaluar la confiabilidad intra e interexaminador de la evaluación de los movimientos mandibulares utilizando el calibre. Métodos: Se trata de un estudio transversal y comparativo realizado en individuos entre 18 y 25 años con síntomas leves o nulos de TTM según el ProDTMMulti. La recolección se realizó en dos días de medición de los movimientos mandibulares de apertura, protrusión, lateralidad derecha e izquierda de cada examinado mediante un calibre plástico analógico. El análisis de concordancia se calculó utilizando el coeficiente de correlación intraclase (CCI), donde se adoptó un nivel de significancia del 5\%. Resultados y discusión: El calibre es un instrumento confiable y tiene una excelente confiabilidad intra-examinador para casi todos los movimientos, excepto para la desviación lateral izquierda, que demostró confiabilidad moderada y la confiabilidad entre examinadores fue moderada para la mayoría de los movimientos. Conclusión: Se puede concluir que el calibre es un método confiable para análisis intra-examinador, sin embargo, esto no ocurre para evaluaciones interexaminador, donde tiene confiabilidad moderada, además de ser muy útil para aplicación clínica que involucre a uno o más examinadores durante el servicio.

Palabras clave: Síndrome de disfunción de la articulación temporomandibular; Prueba de reproducibilidad; Evaluación.

\section{Introdução}

O sistema estomatognático é um sistema anatômico que compreende a mandíbula e maxila, língua, lábios, dentes e tecidos moles associados (glândulas salivares, suprimento nervoso e vascular), articulação temporomandibular (ATM) e músculos mastigatórios (Yoshida, 2020; Barbosa \& Barbosa, 2009). Alterações no nesse sistema estão comumente relacionadas à propriocepção, estabilização visual e estabilidade postural (Manzoti et al., 2020).

A ATM é considerada a articulação mais complexa do organismo, capaz de realizar movimentos altamente coordenados e complexos responsáveis pelas principais funções deste sistema que são mastigação, deglutição, fonação e fala (Lima et al., 2020; Richene \& Cordeiro, 2019; Silva et al., 2011). Para que estes movimentos e funções ocorram de forma adequada e eficiente é importante a ação normal da musculatura facial, cervical e mastigatória, que é responsável pelos movimentos mandibulares de abertura da boca, protrusão, retrusão e desvio lateral (Silva et al., 2012).

Segundo Sassi et al. (2011), a amplitude dos movimentos mandibulares relaciona-se com a integridade da ATM e ação dos músculos, que quando sobrecarregados, excede, a tolerância estrutural e funcional da articulação, podendo levar á disfunção temporomandibular (DTM). As DTMs, condições musculoesqueléticas que afetam tecidos os tecidos envolvidos nos movimentos mandibulares (Martins et al., 2021; Ohrback \& Dworkin, 2019) são a segunda condição musculoesquelética mais comum após a dor lombar crônica, baseado em dados do Instituto Nacional de Pesquisas Odontológicas e Craniofaciais (Manzotti et al., 2020), onde a limitação da amplitude de movimento e os desvios mandibulares são alguns dos principais sintomas (Ribeiro et al., 2011; Viana et al., 2015).

Durante o exame clinico, o conhecimento das limitações dos movimentos mandibulares representa um importante sinal para a avaliação e compreensão do diagnóstico de alterações disfuncionais como a DTM (Benevides et al., 2015; Benevides et al., 2016). Contudo, existem diversos fatores que podem variar os níveis de normalidade dos movimentos 
mandibulares como sexo, idade, altura, peso corpóreo, comprimento do ramo mandibular, frouxidão ligamentar, sobrecarga da ATM e desenvolvimento neuromotor (Benevides et al., 2016).

Atualmente, um dos principais instrumentos utilizados por odontólogos, fonoaudiólogos e fisioterapeutas para avaliação e mensuração dos movimentos mandibulares é o paquímetro. Este é um instrumento, não invasivo, de baixo custo, além de ser de fácil utilização, de precisão e oferece medidas objetivas na avaliação destes movimentos (Pasinato et al., 2011; Biasotto-Gonzalez et al., 2008; Rodrigues et al., 2003).

Segundo Benevides et al. (2015) e Santos \& Vidor (2015) o paquímetro é considerado uma ótima ferramenta para a avaliação do estado funcional do sistema estomatognático, bem como na avaliação de medidas orofaciais pela mensuração da distância entre diferentes pontos da face. Contudo, alguns cuidados devem ser tomados para tornar as medidas mais confiáveis, como a realização das medidas por um único examinador e a recolocação do paquímetro na posição inicial (zero) após cada medição (Rodrigues et al., 2003).

Apesar de Rodrigues et al. (2003) afirmarem que as medidas devem ser realizadas por um único examinador, foram encontrados poucos estudos na literatura que avaliaram a confiabilidade intra e interexaminadores do paquímetro, diferentemente de outros métodos que avaliam amplitude de movimento (ADM) como a goniometria (Venturini et al., 2006; Nonnenmacher, 2021) e a fotogrametria (Di Fonzo et al., 2021; Nonnenmacher, 2021), que já possuem confiabilidade averiguada. Diante disso, o estudo teve como objetivo avaliar a confiabilidade intra e interexaminadores do paquímetro analógico de plástico na avaliação dos movimentos mandibulares.

\section{Métodos}

O presente estudo tem um delineamento transversal e de caráter comparativo (Pereira et al., 2018), e foi aprovado pelo comitê de ética em pesquisa do Hospital Universitário de Aracaju, Universidade Federal de Sergipe (UFS) sob parecer $n^{\circ}$ 829.037/2014. Os voluntários foram devidamente esclarecidos sobre os objetivos e procedimentos realizados e assinaram o Termo de consentimento livre e esclarecido.

Os critérios de inclusão foram idade entre 18 a 25 anos, grau leve ou sem sinais e sintomas de DTM de acordo com o protocolo validado em populações brasileiras para Centros Multiprofissionais na determinação dos sinais e sintomas da DTM (ProDTMMulti) (Felício, Melchior \& Silva, 2009). Os critérios de exclusão foram o uso de aparelho ortodôntico fixo ou móvel, história de doenças sistêmicas como artrite reumatoide e ausência dos incisivos centrais superiores ou inferiores.

Dois examinadores distintos avaliaram os movimentos mandibulares por meio do paquímetro analógico de plástico Disma ${ }^{\circledR}$ graduado em milímetros (Figura 1). Os mesmos seguiram as mesmas condições de avaliação realizando três mensurações para cada movimento e essas medidas foram repetidas em um segundo dia, com intervalo mínimo de 48 horas. Cabe salientar que o conhecimento dos valores das medidas ficou apenas sob o conhecimento do examinador, e somente ao final de toda a coleta, os dados dos dois examinadores foram confrontados. 
Figura 1: Paquímetro analógico de plástico Disma ${ }^{\circledR}$ graduado em milímetros.

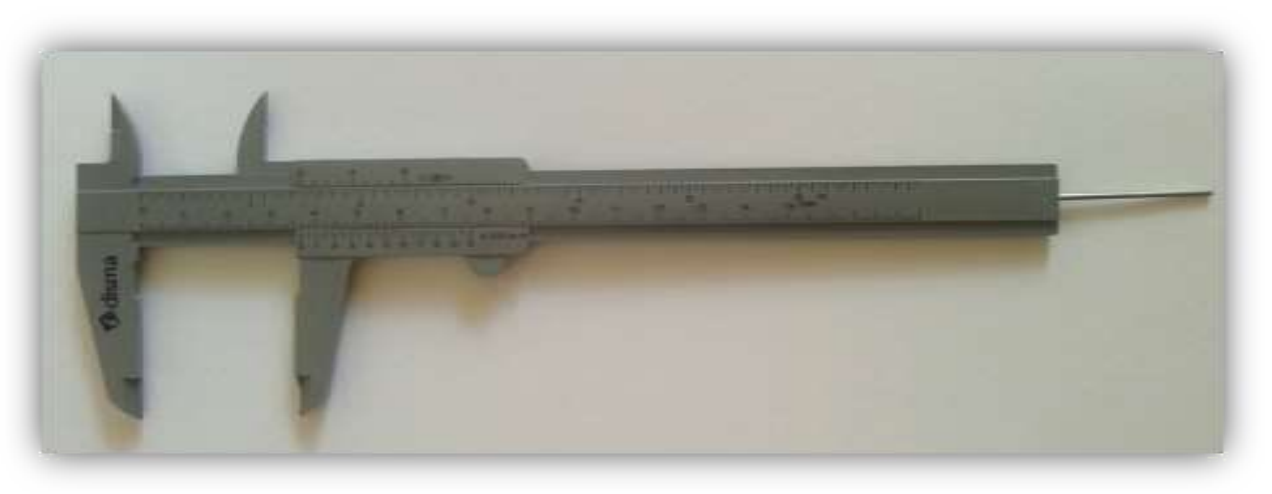

Fonte: Dados dos pesquisadores (2021).

Foram mensurados os principais movimentos mandibulares que, segundo Cattoni (2008) são, abertura da boca, desvio lateral (direita e esquerda) e protrusão. Segundo Barbosa \& Barbosa (2009) e Biasotto-Gonzalez (2005), a abertura da boca é a distância entre as faces incisais dos dentes incisivos superiores e inferiores, acrescidas da medida do trespasse vertical ou overbite, em que se solicita ao indivíduo fazer abertura da boca, posiciona-se a orelha fixa do paquímetro na coroa dentária superior e a orelha móvel na coroa dentária inferior obtendo os valores em milímetros. Para Barbosa \& Barbosa (2009) e Biasotto-Gonzalez (2005), a protrusão é a somatória da medida do trespasse horizontal ou overjet com a medida do deslizamento horizontal da medida sendo feita com a haste do paquímetro encostada entre os dentes incisivos superiores, pedindo para o indivíduo fazer a protrusão máxima.

Barbosa \& Barbosa (2009) afirmam que o desvio lateral (direito/esquerdo) é a medida do deslizamento lateral da mandíbula, registrada da linha entre os incisivos centrais inferiores à linha entre os incisivos centrais superiores instruindo o indivíduo a fazer o desvio lateral, onde a orelha fixa é posicionada na linha entre os incisivos superiores e a orelha móvel na linha entre os incisivos inferiores. A linha mediana foi avaliada com os dentes em oclusão, verificando se as linhas entre os incisivos centrais superiores e inferiores coincidiam (Benevides et al., 2016).

Durante a realização da mensuração, o indivíduo permaneceu em ambiente silencioso, sentado em uma cadeira confortável, adotando uma postura ereta com as plantas dos pés apoiadas no chão e os braços apoiados sobre as pernas, tendo o plano de Frankfurt como parâmetro de posicionamento (Barbosa \& Barbosa, 2009). Cada indivíduo realizou a amplitude máxima de movimento em todos os movimentos e antes da mensuração, os indivíduos tinham que fazer os movimentos pedidos sob orientação dos examinadores.

Para analisar a confiabilidade inter e intraexaminadores, foram calculados o Coeficiente de Correlação Intraclasse (ICC) (Snedecor \& Cochran, 1972). O ICC permite medir o grau de concordância entre as medidas dentro de uma classe. De acordo com Fleiss, Levin \& Paik (2003) a confiabilidade é considerada pobre quando for $<0,4$; moderada quando for de 0,4 a 0,75; e excelente quando for $>0,75$. Quanto mais próximo o coeficiente estiver de 1 , mais concordância haverá entre as medidas dentro de uma mesma classe, adotando para todas as análises um nível de significância de 5\%.

\section{Resultados e Discussão}

Os resultados foram estudados por meio de uma amostra constituída de 31 voluntários que se dispuseram a realizar a pesquisa. Os voluntários foram discentes da UFS, Campus universitário Professor Antônio Garcia Filho (Lagarto - SE), dos cursos de fisioterapia, farmácia, fonoaudiologia e odontologia, de ambos os gêneros, sendo cinco do sexo masculino e 26 do sexo feminino, com idade média de 22,5 anos. 
A tabela 1 ilustra os resultados da confiabilidade interexaminadores obtidos no primeiro e segundo dia de avaliação dos movimentos mandibulares. É possível notar que dentre as variáveis analisadas os movimentos de abertura, protrusão e desvio lateral direito apresentaram moderada confiabilidade de acordo com a classificação do ICC no primeiro dia de mensuração e o movimento de desvio lateral esquerdo apresentou uma confiabilidade pobre. No segundo dia, a abertura apresentou uma excelente confiabilidade, já que os outros movimentos apresentaram-se com mesmos resultados do primeiro dia.

Tabela 1: Análise da concordância interexaminadores dos movimentos mandibulares por meio do paquímetro realizada em dois dias de mensuração

\begin{tabular}{|c|c|c|c|c|}
\hline Dia de mensuração & Variável & ICC & Intervalo de confiança (95\%) & Classificação \\
\hline \multirow{6}{*}{1} & Abertura & 0,52 & 0,$21 ; 0,74$ & Moderada \\
\hline & Protrusão & 0,61 & 0,$34 ; 0,79$ & Moderada \\
\hline & Desvio Lateral & & & Moderada \\
\hline & Direito & 0,58 & 0,$29 ; 0,77$ & \multirow{3}{*}{ Pobre } \\
\hline & Desvio Lateral & & & \\
\hline & Esquerdo & 0,27 & $-0,09 ; 0,56$ & \\
\hline \multirow{6}{*}{2} & Abertura & 0,77 & 0,$58 ; 0,88$ & Excelente \\
\hline & Protrusão & 0,71 & 0,$48 ; 0,85$ & Moderada \\
\hline & Desvio Lateral & & & Moderada \\
\hline & Direito & 0,45 & 0,$12 ; 0,69$ & \multirow{3}{*}{ Pobre } \\
\hline & Desvio Lateral & & & \\
\hline & Esquerdo & 0,33 & $-0,03 ; 0,61$ & \\
\hline
\end{tabular}

Legenda: ICC = Intervalo de Correlação Intraclasse.

$1=$ Primeiro Dia de Avaliação

2 = Segundo Dia de Avaliação

Fonte: Dados dos pesquisadores (2021).

Os dados obtidos na análise de concordância intraexaminadores está ilustrado na tabela 2. Nesta, pode-se notar que dentre as variáveis analisadas apenas o movimento de desvio lateral esquerdo apresentou uma confiabilidade moderada pelo examinador 1 e os outros movimentos analisados apresentaram uma excelente confiabilidade para ambos examinadores.

Tabela 2: Análise da concordância intraexaminador dos movimentos mandibulares por meio do paquímetro realizada por diferentes examinadores.

\begin{tabular}{lllcc}
\hline Avaliador & Variável & ICC & Intervalo de confiança (95\%) & Classificação \\
\hline \multirow{5}{*}{ Examinador 1 } & Abertura & 0,88 & 0,$77 ; 0,94$ & Excelente \\
& $\begin{array}{l}\text { Protrusão } \\
\text { Desvio Lateral } \\
\text { Direito }\end{array}$ & 0,90 & 0,$80 ; 0,95$ & $\begin{array}{l}\text { Excelente } \\
\text { Excelente }\end{array}$ \\
& $\begin{array}{l}\text { Desvio Lateral } \\
\text { Esquerdo }\end{array}$ & 0,84 & 0,$70 ; 0,92$ & Moderada \\
\hline \multirow{5}{*}{ Examinador 2 } & Abertura & 0,78 & 0,$41 ; 0,82$ & Excelente \\
& $\begin{array}{l}\text { Protrusão } \\
\text { Desvio Lateral } \\
\text { Direito } \\
\text { Desvio Lateral } \\
\text { Esquerdo }\end{array}$ & 0,89 & 0,$60 ; 0,89$ & Excelente \\
& 0,91 & 0,$78 ; 0,94$ & Excelente \\
& 0,93 & 0,$82 ; 0,95$ & Excelente \\
\hline
\end{tabular}

Legenda: ICC = Intervalo de Correlação Intraclasse.

Fonte: Dados dos pesquisadores (2021). 
Atualmente as pesquisas que utilizam medidas físicas para a mensuração de dados clínicos necessitam de uma avaliação e precisão na validade e confiabilidade do instrumento que está sendo utilizado. Quando uma medida é avaliada, pode haver erros relacionados ao examinador e/ou ao instrumento usado, o que pode gerar vieses ou aumento da imprecisão no estudo e prática clínica (Santos et al., 2011; Artese et al., 2012).

Estabelecer a confiabilidade de instrumentos que são utilizados na prática clínica é de fundamental importância para o examinador. Para isto, sabe-se que a utilização dos mesmos deve-se passar por treinamento para evitar falhas que possam comprometer a pesquisa ou o plano de tratamento. Sendo assim, é importante entender que a confiabilidade é um dos principais parâmetros que assegura o profissional a precisão quantitativa e qualitativa do tratamento de seus pacientes (Venturini et al., 2006; Artese et al., 2011).

O paquímetro é um instrumento bastante utilizado na mensuração dos movimentos mandibulares, e apesar de ser um instrumento simples, não invasivo e de fácil utilização, é sabido que sua confiabilidade ainda é pouco estudada. Inúmeras são as vantagens da utilização do paquímetro analógico de plástico, dentre elas está o fato de ser acessível financeiramente, apresentar facilidade de manipulação após breve familiarização do seu uso, ser de fácil quantificação e armazenamento das informações e ser um facilitador da comunicação entre os profissionais das áreas de fisioterapia, odontologia e fonoaudiologia (Benevides et al., 2016; Rodrigues et al., 2003; Oliveira et al., 2007).

Nesse estudo, ao analisar os movimentos mandibulares por meio do paquímetro, pode-se dizer que a confiabilidade intraexaminadores (Tabela 02) foi excelente, com exceção do desvio lateral esquerdo no examinador 1. Já a confiabilidade interexaminadores (Tabela 01) foi, em maior parte, considerada moderada para ambos os dias de mensuração, exceto no segundo dia de avaliação em que a abertura foi considerada excelente e em ambos os dias em que a confiabilidade do desvio lateral esquerdo foi considerada pobre.

O único estudo encontrado na literatura que avalia a confiabilidade intra e interexaminador é o de Rodrigues et al. (2008), por meio do paquímetro digital nas medidas mandibulares de abertura máxima de boca. Eles verificaram que os resultados obtidos intraexaminador demostraram um valor $(\mathrm{p}=0,0677)$ e interexaminador $(\mathrm{p}<0,0001)$. $\mathrm{O}$ que não corrobora com o presente estudo, em que o movimento de abertura da boca apresentou uma confiabilidade intraexaminador excelente.

Já o estudo de Best et al. (2013) analisou os movimentos mandibulares de 30 indivíduos saudáveis e 30 indivíduos com DTM, e mensurou os movimentos de abertura, lateralidade, protrusão, overbite e overjet por meio do paquímetro analógico de plástico e repetiu essas medidas após sete dias da primeira avaliação. Eles calcularam a confiabilidade intra e intersessão de um mesmo examinador e a interexaminadores. Para a intraexaminadores, obtiveram como resultado uma confiabilidade excelente, assim como o presente estudo. Para interexaminadores eles também tiveram como resultado uma excelente confiabilidade, o que não corrobora com esta pesquisa, já que a mesma obteve moderada confiabilidade, na maior parte dos movimentos. Sendo assim, percebe-se que ainda é preciso estudos que visem identificar esta confiabilidade do uso do paquímetro na avaliação dos movimentos mandibulares.

Existem fatores que podem interferir nas medições, como questões psicológicas que são responsáveis por alterações neuromusculares do sistema estomatognático e o estresse que causa aumento na atividade muscular gerando espasmo e fadiga, estando associado ao surgimento de atividades parafuncionais orais (Paulino et al., 2018; Quinto, 2000). A realização das medidas em diferentes dias também pode interferir nos resultados, pois a flexibilidade muscular pode variar e alterar o resultado obtido, como também pode alterar devido as diferentes possibilidades de manuseio do paquímetro (OLIVEIRA et al., 2007).

Pode-se destacar que como a confiabilidade intraexaminador deu excelente para a maior parte dos movimentos, e interexaminador moderada a pobre, nem os fatores psicológicos, estresse ou variações de flexibilidade muscular influenciaram nas medidas obtidas, mas as diferentes possibilidades de manuseio do paquímetro pelos examinadores, como sugerido por 
Oliveira et al. (2007), podem ter influenciado o resultado do presente estudo. No entanto, os avaliadores observaram que os fatores podem ter influenciado os resultados, como a falta de habilidade dos voluntários em realizar o movimento de desvio lateral direito e esquerdo. Bem como a inexperiência das avaliadoras que foram treinadas em um período curto.

O goniômetro é um método muito utilizado na fisioterapia para medir ADM das articulações, com exceção da ATM, na avaliação e reavaliação de pacientes com diversos problemas osteomioarticulares (Amoras et al., 2021; Herrero et al., 2011). Diversos estudos já atestaram a confiabilidade desse método, como Herrero et al. (2011), Otter et al. (2015) e Gouveia et al. (2014) que apresentaram confiabilidade intraexaminador excelente e interexaminador moderada a excelente do goniômetro. Comprova-se que, assim como o goniômetro, o paquímetro apresenta resultado de confiabilidade intraexaminador excelente. Pitangui et al. (2016) e Belo (2016) também classificaram o paquímetro como um instrumento confiável e com concordância em seus estudos.

Por fim, é importante destacar que para avaliação, acompanhamento e reavaliação de pacientes com DTM e outras desordens do sistema estomatognático devem ser utilizados instrumentos no qual diferentes profissionais como fisioterapeuta, fonoaudiólogo e odontólogo possam utilizar com confiança no planejamento e aplicação de condutas terapêuticas no tratamento dos pacientes (Deda, 2013).

\section{Conclusão}

A utilização do paquímetro analógico de plástico na avaliação dos movimentos mandibulares vem sendo bastante utilizada na prática clínica, no entanto, sua confiabilidade para esses movimentos tem sido pouco testada. O presente estudo permite observar que este instrumento apresenta uma excelente confiabilidade intraexaminador para quase todos os movimentos, exceto desvio lateral esquerdo que demonstrou uma moderada confiabilidade e em sua maioria uma confiabilidade moderada interexaminadores. Demonstrando assim, ser um método de grande utilidade para a aplicação clínica, durante o atendimento e acompanhamento de pacientes com disfunções do sistema estomatognático na rotina do fisioterapeuta, fonoaudiólogo e/ou odontólogo.

Sugere-se a elaboração de futuros estudos, com diferentes populações, para avaliar as disfunções do sistema estomatognático com a utilização do paquímetro analógico e preencher as demais lacunas presentes na literatura.

\section{Referências}

Amoras, R. M., Lima, R. R. P., Felix, G. T., \& Freitas, W. M. T. M. (2021). Efeitos de um protocolo fisioterapêutico para tratamento de paciente com disfunção temporomandibular: um relato de caso. Inova Saúde, 11(2): 189-199.

Artese H. P. C., Rabelo M. S., Foz, A. M., Horliana, A. C. R. T., Romito, G. A., \& Pannuti, C. M. (2012). Como estabelecer confiabilidade e evitar erros de mensuração nas pesquisas clínicas periodontais?. Braz J Periodontol., 22(2):36-40.

Barbosa, V. C. S., \& Barbosa, F. S. (2009). Fisioterapia nas disfunções temporomandibulares. São Paulo, SP: Phorte.

Belo, M. C. F. (2016). Confiabilidade interavaliadores do paquímetro na mensuração da distância inter-retos e sua acurácia no diagnóstico da diástase dos músculos retos do abdome no terceiro trimestre gestacional. Dissertação de Mestrado. Universidade Federal de Pernambuco, Recife, PE, Brasil.

Benevides, A. D., Araujo, R. P., Garcia, D. G. B., Ribeiro, C. O., \& Mello, A. M. F. (2014) Fatores que interferem na amplitude dos movimentos mandibulares em crianças. Rev. CEFAC, 16(1):237-243. DOI:10.1590/1982-0216201425512.

Benevides, S. D., Araujo, R. P., Ribeiro, C. O., \& Mello, S. M. F. (2016). Determinação da amplitude dos movimentos mandibulares em crianças do estado da Bahia. Rev. CEFAC, 18(1):95-103. DOI: 10.1590/1982-0216201618110515.

Best, N., Best, S., Loudovici-Krug, D., \& Smonlenski, U. C. (2013). Measurement of mandible movements using a vernier caliper na evaluation of the intrasesssion, intersession and interobserver realiability. CRANIO®, 31(3):176-180. DOI: 10.1179/crn.2013.028.

Biasotto-Gonzalez, D. A., Andrade, D. V., Gonzalez, T. O., Martins, M. D., Fernandes, K. P. S., Corrêa, J. C. F., \& Bussadori, S. K. (2008). Correlação entre disfunção temporomandibular, postura e qualidade de vida. Rev Bras Crescimento Desenvolv. Hum., 18(1):79-86.

Biasotto-Gonzalez, D. A. (2005). Abordagem interdisciplinar das disfunções temporomandibulares. Barueri, SP: Manole. 
Cattoni, D. M., Fernandes, F. D. M., Di Francesco, E. C., \& Latorre, M. R. D. O. (2008). Medidas e proporções antropométricas orofaciais de crianças respiradoras orais. Rev Soc Bras Fonoaudiol., 13(2):119-26. DOI: 10.1590/S1516-80342008000200005.

Deda, M. R. C. (2013). Efeito do tratamento da deformidade dentofacial nos sinais e sintomas de DTM, na postura de cabeça e na atividade eletromiográfica [tese]. Universidade de São Paulo, Faculdade de Medicina de Ribeirão Preto, Ribeirão Preto, SP, Brasil.

Felício, C. M., Melchior, M. O., \& Silva, M. A. M. R. (2009). Clinical Validity of the Protocol for MultiProfessional Centers for the Determination of Signs and Symptoms of Temporomandibular Disorders Part II. The Journal of craniomandibular practice, 27(1):62-67. DOI: 10.1179/crn.2009.010.

Fleiss, J. L., Levin, B., \& Paik, M. C. (2003). Statistical Methods for Rates and Proportions. Hoboken. New Jersey: John Wiley \& Sons. Inc.

Fonzo, A. L. D., Pinto, H. G., Rabelo, I. J., Elchin, C. B., Cometti, G. F., Bezerra V., Pinho, J. P. S. F. M., Guerra, K., Coto, N. P., \& Dias, R. B. (2021). Study of the athlete's posture and balance through photogrammetry and stabilometry when using mouthguards for sports. RSD [Internet], 10(9):e11510917730. DOI: $10.33448 /$ rsd-v10i9.17730.

Gouveia, V. H. O., Araújo, A. G. F., Maciel, S. S., Ferreira, J. J. A., \& Santos, H. H. (2014). Confiabilidade das medidas inter e intra-avaliadores com goniômetro universal e flexímetro. Fisioter Pesq., 21(3):229-35. DOI: 0.590/1809-2950/52921032014.

Herrero, P., Carrera, P., García, E., Gómez-Trullén, E. T., \& Oliván-Blázquez, B. (2011). Reliability of goniometric measurements in children with cerebral palsy: A comparative analysis of universal goniometer and electronic inclinometer: A pilot study. BMC Musculoskeletal Disorders, 12(1):1-8. DOI: $10.1186 / 1471-2474-12-155$.

Lima, L. F. C., Silva, F. A. de J. C., Monteiro, M. H. A., \& Oliveira Júnior, G. (2020). Depression and anxiety and association with temporomandibular disorders - literature review. Research, Society and Development, 9(7), e579974540. DOI: 10.33448/rsd-v9i7.4540

Manzotti, A., Viganoni, C., Lauritano, D., Bernasconi, S., Paparo, A., Risso, R., \& Nanussi, A. (2020). Evaluation of the Stomatognathic System before and after Osteopathic Manipulative Treatment in 120 Healthy People by Using Surface Electromyography. International journal of environmental research and public health, 17(9), 3250. DOI: 10.3390/ijerph17093250.

Martins, S. S., Santana, L. N., Rodrigues Nascimento, V., Moreira Rocha, L., Araujo Cruz, J. W., Maciel, L. Y. dos S., Paiva, S. F. de., \& Costa, M. R. de C. D. (2021). Conhecimento de profissionais a respeito da disfunção temporomandibular: um estudo piloto. RECIMA21 - Revista Científica Multidisciplinar ISSN 2675-6218, 2(7), e27530. DOI: 10.47820/recima21.v2i7.530

Nonnenmacher, C. H. (2021). Confiabilidade intra e interavaliador para medidas angulares de membro inferior através da fotogrametria. Trabalho de Conclusão de Curso. Universidade Federal de Santa Catarina, Araranguá, SC, Brasil.

Ohrbach, R., \& Dworkin, S. F. (2019). AAPT Diagnostic Criteria for Chronic Painful Temporomandibular Disorders. The journal of pain, 20(11), 1276-1292. DOI: $10.1016 /$ j.jpain.2019.04.003.

Oliveira, D. D., Ruellas, A. C. O., Drummond, M. E. L., Pantuzo, M. C. G., \& Lanna, A. M. Q. (2007). Confiabilidade do uso de modelos digitais tridimensionais como exame auxiliar ao diagnóstico ortodôntico: um estudo piloto. R Dental Press Ortodon Ortop Facial, 12(1):84-93. DOI: 10.1590/S141554192007000100012

Otter, S. J., Agalliu, B., Baer, N., Hales, G., Harvey, K., James, K., Keating, R., McConnell, W., Nelson, R., Qureshi, S., Ryan, S., St John, A., Waddington, H., Warren, K., \& Wong, D. (2015). The reliability of a smartphone goniometer application compared with a traditional goniometer for measuring first metatarsophalangeal joint dorsiflexion. Journal of foot and ankle research, 8, 30. DOI: 10.1186/s13047-015-0088-3

Pasinato, F., Souza, J. A., Corrêa, E., \& Silva, A. (2011). Temporomandibular disorder and generalized joint hypermobility: application of diagnostic criteria. Brazilian journal of otorhinolaryngology, 77(4), 418-425. DOI: 10.1590/S1808-86942011000400003.

Paulino, M. R., Moreira V.G., Lemos, G. A., Silva P. L. P., Bonan. P.R. F., \& Batista, A. U. D. (2018). Prevalência de sinais e sintomas de disfunção temporomandibular em estudantes pré-vestibulandos: associação de fatores emocionais, hábitos parafuncionais e impacto na qualidade de vida. Ciência \& Saúde Coletiva, 23(1): 173-186. DOI: 10.1590/1413-81232018231.18952015

Pereira, AS, Shitsuka, DM, Parreira, FJ \& Shitsuka, R. (2018). Metodologia da pesquisa científica. [e-book]. Santa Maria. Ed. UAB/NTE/UFSM. Disponível em: https://repositorio.ufsm.br/bitstream/handle/1/15824/Lic_Computacao_Metodologia\%20PesquisaCientifica.pdf?sequence=1.

Pitangui, A. C. R., Fukagawa, L. K., Barbosa, C. S., Lima, A. S., Gomes, M. R. de A., \& Araújo, R. C. de. (2016). Analysis of reliability and agreement of the methods caliper and fingerbreadths to measure the rectus abdominis diastasis. ABCS Health Sciences, 41(3). DOI: 10.7322/abcshs.v41i3.904.

Quinto, C. A. (2000) Classificação e tratamento das disfunções temporomandibulares: qual o papel do fonoaudiólogo no tratamento dessas disfunções?. Rev CEFAC, 2(2):15-22.

Ribeiro, B. G., Costa, J. M., Gomes, C. A. F. P., Hage, Y., Arruda, E. E. C., Gonzalez, T. O., Politti, F., \& Biasotto-Gonzalez, D. A. (2011). Avaliação do movimento mandibular em crianças com e sem disfunção temporomandibular. ConScientiae Saúde, 10(4):729-34. DOI: 10.5585/ConsSaude.v10i4.3040

Richene, R. V., \& Cordeiro, R. S. (2019). Atuação Fisioterapeutica Nas Disfunções Da Articulação Temporomandibular. Journal of Specialist, 1(3).

Rodrigues, F. V., Monção, F. R. C., Moreira, M. B. R., \& Motta, A. R. (2008). Variabilidade na mensuração das medidas orofaciais. Rev. Soc. Bras. Fonoaudiol.,13(4):332-7. DOI: 10.1590/S1516-80342008000400006.

Rodrigues, K. A., Lefévre, A. P., Mott, L. B., Tugumia, D., \& Peña, P. L. (2003). Análise comparativa entre o lado de predominância mastigatória e medidas da mandíbula por meio do paquímetro. Rev CEFAC, 5(4):347-351.

Santos, J. D. M., Oliveira, M. A., Silveira, N. J. F., Carvalho, S. S., \& Oliveira, A. G. (2011). Confiabilidade inter e intraexaminadores nas mensurações angulares por fotogrametria digital e goniometria. Fisioter Mov., 24(3):389-400. DOI: 10.1590/S0103-51502011000300003. 
Research, Society and Development, v. 10, n. 13, e190101321049, 2021

(CC BY 4.0) | ISSN 2525-3409 | DOI: http://dx.doi.org/10.33448/rsd-v10i13.21049

Santos, K. W., \& Vidor, D. C. G. M. (2015). Medidas faciais em indivíduos adultos sem queixas orofaciais: compatibilidade entre medidas antropométricas e percepção facial. Rev. CEFAC, 17(1):126-133. DOI: 10.1590/1982-021620154014.

Santos, L. H. G. (2012). Avaliação funcional da disfunção temporomandibular após bioestimulação associado í cinesioterapia. Fisioterapia Brasil. 13(4): 264271.

Sassi, F. C., Mangilli, L. D., Poluca, M. C., Bento, R. F., \& Andrade, C. R. F. (2011). Mandibular range of motion in patients with idiopathic peripheral facial palsy. Braz J Otorhinolaryngol., 77(2):237-44. DOI: 10.1590/s1808-86942011000200014.

Snedecor, G. W., \& Cochran, W. G. (1972) Statistical Methods, Sixth Edition. Iowa: The Iowa State University Press.

Venturini, C., Ituassú, N. T., Teixeira, L. M., \& Deus, C. V. O. (2006). Confiabilidade intra e interexaminadores de dois métodos de medida da amplitude ativa de dorsiflexão do tornozelo em indivíduos saudáveis. Rev. bras. fisioter., 10(4):407-11. DOI: 10.1590/S1413-35552006000400008.

Viana, M. O., Lima, E. I. C. B. M. F., Menezes, J. N. R., \& Olegario, N. B. C. (2015). Avaliação de sinais e sintomas da disfunção temporomandibular e sua relação com a postura cervical. Rev Odontol UNESP, 44(3): 125-130. DOI: 10.1590/1807-2577.1071.

Yoshida, K. (2020). Clinical Characteristics of Functional Movement Disorders in the Stomatognathic System. Front Neurol., 11:123. DOI: 10.3389/fneur.2020.00123. 